\title{
SOPRA I GRUPPI DEFINITI DA EQUAZIONI DIFFERENZIALI DEL PRIMO ORDINE.
}

\author{
Nota di Paolo Medolaghi (Roma).
}

Adunanza del 9 giugno 1907.

In una Nota pubblicata qualche anno $\mathrm{fa}^{\mathrm{r}}$ ), occupandomi del problema della determinazione dei gruppi continui, finiti ed infiniti, in $n$ variabili, mostrai in base a quali procedimenti la ricerca poteva, almeno teoricamente, essere semplificata. In quella occasione enunciai un teorema generale che riconduce la determinazione dei gruppi transitivi a quella dei gruppi che contengono tutte le traslazioni (gruppi di PICARD, perchè fu questo il primo Autore che se ne occupó in modo particolare nei suoi studî sulla generalizzazione delle funzioni di variabile complessa).

Il Prof. Vessiot, in una Memoria ${ }^{2}$ ) nella quale riprende, completa e generalizza le ricerche di ENGEL e mie sull'argomento, ha giustamente osservato come la dimostrazione da me data in quella occasione non sia completa. Egli considera il gruppo infinito in tre variabili che lascia invariante un sistema completo, e, determinando le condizioni di integrabilita del sistema delle equazioni di definizione, scritte sotto la forma canonica, perviene ad una condizione di un tipo speciale che io non avevo considerata tra le possibili condizioni di integrabilità.

Nella presente Nota io mi propongo anzitutto di completare la mia antica dimostrazione, per il caso di gruppi definiti da sole equazioni differenziali del primo ordine (gruppi del primo ordine). Quando si prescinda dal gruppo proiettivo generale, e da quello infinito delle trasformazioni proporzionali, che sono definiti da sole equazioni del secondo ordine, ogni altro gruppo sia finito, sia infinito, o è del primo ordine, o è contenuto in un gruppo del primo ordine.

Sopra il teorema di esistenza dei gruppi di PICARD può essere fondato un indirizzo di ricerche, che sembra promettente, e di cui ho cercato di cogliere i primi, più facili frutti. Il risultato più interessante è il nesso intimo stabilito, nell'ultimo paragrafo, tra questo indirizzo di ricerca, ed il metodo degli sviluppi in serie nell'intorno di un punto generico.

r) Contributo alla determinazione dei gruppi continui in uno spazio ad $n$ dimensioni [Rendiconti della R. Accademia dei Lincei, vol. VIII, ${ }^{\circ}{ }^{\circ}$ semestre I899, pp. 29I.295].

2) Sur la théorie des groupes continus [Annales scientifiques de l'École Normale supérieure, $\mathrm{III}^{\mathrm{e}}$ série, t. XX (1903), pp. 4[1]-45I]. 
Nei primi tre paragrafi, nei quali mi riferisco a lavori precedenti, adotto per comodita dei raffronti, tutte le notazioni del Vessiot.

I. Le equazioni di definizione per le trasformazioni finite di un gruppo $G$ il quale operi sulle $n$ variabili $x_{1}, \ldots, x_{n}$ si possono porre sempre sotto la forma:

(1) $\quad L_{s}\left[\omega_{1}\left(y_{1}, \ldots, y_{n}\right), \ldots, \omega_{p}\left(y_{1}, \ldots, y_{n}\right) \mid \ldots y_{j}^{\left(\delta_{1}, \ldots, \hat{r}_{n}\right)} \ldots\right]=\omega_{s}\left(x_{\mathrm{s}}, \ldots, x_{n}\right) \quad(s=\mathrm{I}, 2, \ldots, p)$ in cui :

$$
y_{j}^{\left(\delta_{1}, \ldots, \hat{\delta}_{n}\right)}=\frac{\partial^{\delta_{1}+\cdots+\delta_{n}} y_{j}}{\partial x_{1}^{\delta_{1}} \ldots \partial x_{n}^{\delta_{n}}}
$$

e le $\omega_{1}, \ldots, \omega_{p}$ sono determinate funzioni delle variabili.

Accanto al sistema (I) torna opportuno considerare quello

$$
L_{s}\left[\omega_{1}\left(y_{1}, \ldots, y_{n}\right), \ldots, \omega_{p}\left(y_{1}, \ldots, y_{n}\right) \mid \ldots y_{j}^{\left(\delta_{1}, \ldots, \delta_{n}\right)} \ldots\right]=J_{s}\left(x_{1}, \ldots, x_{n}\right) \quad(s=1,2, \ldots, p)
$$

che si deduce $\mathrm{da}(\mathrm{I})$ sostituendo nei secondi membri delle equazioni in luogo delle funzioni $\omega_{1}(x), \ldots, \omega_{p}(x)$ rispettivamente le $\tau_{1}(x), \ldots, \jmath_{p}(x)$.

Si dimostra anzitutto che se il sistema (I) è completamente integrabile, e se il sistema (2) è integrabile, allora anche (2) è completamente integrabile. E inversamente, se il sistema (2) è completamente integrabile tale è anche il primitivo sistema (I).

La interpretazione geometrica di queste condizioni è semplicissima. Che il sistema (2) sia integrabile è la condizione necessaria e sufficiente affinchè i due gruppi che corrispondono l'uno al sistema di funzioni $\omega_{1}, \ldots, \omega_{p}$, l'altro al sistema $\Xi_{1}, \ldots, J_{p}$ siano simili tra loro.

Il sistema (2) sarà o non sarà integrabile secondo la natura delle funzioni $\omega_{1}, \ldots, \omega_{p}, \Im_{1}, \ldots, \Im_{p}$; infatti se noi andiamo a scrivere le condizioni di integrabilita si trovano in ultima analisi delle relazioni differenziali a cui devono soddisfare le funzioni suddette. Una prima importante osservazione ci permette fin dal principio di stabilire che, quando il nostro studio sia limitato ai gruppi transitivi, nessuna relazione di ordine zero, cioè in termini finiti, puó esistere tra le funzioni $\omega_{1}, \ldots, \omega_{p}$ e le $\nexists_{1}, \ldots, \nexists_{p}$ (o fra le sole $\omega$ e le sole $\Im$ ).

Si dimostra poi, fondandosi sulla natura speciale delle funzioni $L_{1}, \ldots, L_{p}$, che la ricerca delle condizioni di integrabilità per il sistema (2) equivale alla ricerca delle condizioni di equivalenza di due varietà rispetto ad un gruppo. Si tratta infatti di esprimere che le varietà :

ed

$$
\begin{array}{ll}
u_{s}=\omega_{s}\left(x_{1}, \ldots, x_{n}\right) & (s=1, \ldots, p) \\
u_{s}=z_{s}\left(x_{1}, \ldots, x_{n}\right) & (s=1, \ldots, p)
\end{array}
$$

sono equivalenti tra loro, rispetto al gruppo la cui trasformazione infinitesima generica è :

$$
\Xi F=\sum_{i=1}^{n} \xi_{i} \frac{\partial F}{\partial x_{i}}+\sum_{i \mid \alpha_{1}, \ldots, \alpha_{n}} \frac{\xi_{i}^{\left(\alpha_{1}, \ldots, \alpha_{n}\right)}}{\alpha_{1} ! \ldots ! \alpha_{n} !} \Lambda_{i \mid \alpha_{1}, \ldots, \alpha_{n}} F
$$

Secondo la teoria generale dell'equivalenza le condizioni che si dovranno trovare sono di due specie; le une esprimono che le varietd equivalenti tra loro debbono eventualmente appartenere allo stesso sistema differenziale invariante rispetto al gruppo (3) 
esteso; le altre esprimono che gli invarianti differenziali di questo gruppo debbono assumere nei punti omologhi delle due varietà lo stesso valore numerico.

Si trovano dunque dei sistemi di equazioni della forma generale ${ }^{3}$ ):

$$
\begin{array}{ll}
\Omega_{b}\left(u_{1}, \ldots, u_{p} \mid \ldots \frac{\partial u_{s}}{\partial x_{i}} \ldots\right)=o & (b=\mathrm{r}, 2, \ldots, \rho) \\
I_{k}\left(u_{1}, \ldots, u_{p} \mid \ldots \frac{\partial u_{s}}{\partial x_{i}} \ldots\right)=c_{k} & (k=\mathrm{I}, \ldots, r) .
\end{array}
$$

Il caso in cui non intervengano che equazioni del tipo (5) è stato già da me considerato nella Nota citata in principio; per esso si vede come in generale i gruppi compresi sotto la forma canonica di ENGEL (tipo di ENGEL) si possono distribuire in un numero infinito di classi, ciascuna classe essendo costituita da tutti i gruppi simili tra loro (tipo di LiE). Inoltre tra questi tipi di Lie ve ne è sempre uno caratterizzato dalla circostanza che per esso le funzioni $\omega_{s}$ si riducono a costanti, la qual cosa esprime che nel gruppo entrano tutte le traslazioni.

Per stabilire questo risultato anche nel caso più generale in cui tra le condizioni di integrabilità ve ne siano alcune della forma (4) basterà dimostrare che queste equazioni sono sempre soddisfatte quando si pone

$$
\omega_{\mathrm{I}}=\text { cost., } \ldots, \omega_{p}=\text { cost. }
$$

2. Con procedimenti ben noti si possono trovare tutte le varietà invarianti rispetto a un gruppo finito. Se il gruppo è ad $r$ parametri ed in $n$ variabili, le sue trasformazioni infinitesime indipendenti forniscono $r n$ coefficienti; disponendo questi in una matrice rettangolare ed eguagliando a zero tutti i minori di ordine $r-b+$ I della matrice si ottengono, per ogni valore di $h$, dei sistemi di equazioni che, quando sono compatibili, definiscono varietà invarianti.

Con un procedimento analogo, dopo aver esteso una, due, ... volte il gruppo la cui trasformazione infinitesima generica è la (3) se ne troveranno le varietà invarianti. Tornerà comodo cambiare un poco le notazioni, e invece di dire che il gruppo (3) opera sopra le variabili $x_{1}, \ldots, x_{n}, u_{1}, \ldots, u_{p}$ diremo che opera sopra le $x_{1}, \ldots, x_{n}$, $\omega_{1}, \ldots, \omega_{p}$; estenderemo poi tale gruppo per riguardo alle derivate delle $\omega_{1}, \ldots, \omega_{p}$, rispetto alle $x_{1}, \ldots, x_{n}$ e studieremo le matrici dalle quali si ricavano tutti $i$ possibili sistemi di equazioni invarianti.

La legge generale di formazione di tali matrici sard̀ la seguente: in una stessa colonna si dovranno porre $i$ coefficienti che si incontrano considerando la variazione infinitesima di una determinata variabile $\left(\omega_{1}, \ldots, \omega_{p}, \ldots \frac{\partial \omega_{s}}{\partial x_{i}} \ldots\right)$; in una stessa linea si dovranno porre $i$ coefficienti che hanno per comune fattore una stessa arbitraria (cioè una stessa derivata delle $\xi$ ).

Prima di trattare il caso generale non sarà inopportuno fermarsi a considerarne

3) Equazioni (58) e (59) di Vessior (mem. cit.). 
uno particolare e più semplice. Supponiamo che nella trasformazione infinitesima generica del nostro gruppo (3) non intervengano che le detivate prime delle $\xi_{1}, \ldots, \xi_{n}$ e che si tratti di cercare $\mathrm{i}$ sistemi differenziali invarianti del primo ordine nelle derivate delle $\omega_{1}, \ldots, \omega_{p}$,

Per ipotesi dunque è:

$$
\begin{cases}\Xi x_{i}=\xi_{i} & (i=1, \ldots, n) \\ \Xi \omega_{s}=\sum_{i=1}^{n} \sum_{k=1}^{n} \frac{\partial \xi_{i}}{\partial x_{k}} \alpha_{i k s}\left(\omega_{1}, \ldots, \omega_{p}\right) & (s=1, \ldots, p)\end{cases}
$$

e si ha quindi:

$$
\Xi \frac{\partial \omega_{s}}{\partial x_{h}}=\frac{\partial\left(\boldsymbol{\Xi} \omega_{s}\right)}{\partial x_{b}}-\sum_{i=1}^{n} \frac{\partial \omega_{s}}{\partial x_{i}} \frac{\partial \xi_{i}}{\partial x_{h}} \quad\left(\begin{array}{c}
s=1, \ldots, p \\
b=1, \ldots, n
\end{array}\right) .
$$

Senza che sia necessario sviluppare le derivazioni accennate nei secondi membri di queste ultime relazioni noi vediamo che le derivate delle $\xi$ non vi figurano che fino al secondo ordine, e che esse hanno per coefficienti le funzioni $\alpha_{i k s}$ delle $\omega_{1}, \ldots, \omega_{p}$; le derivate prime delle $\xi$ hanno per coefficienti delle espressioni lineari omogenee nelle derivate delle $\omega_{1}, \ldots, \omega_{p}$.

La matrice cercata puó quindi essere rappresentata schematicamente cosi :

\begin{tabular}{c|c|c|} 
& $\begin{array}{c}\text { variazioni } \\
\text { delle } \omega_{1}, \ldots, \omega_{p}\end{array}$ & $\begin{array}{r}\text { variazioni delle derivate prime } \\
\text { delle } \omega_{1}, \ldots, \omega_{p}\end{array}$ \\
\hline $\begin{array}{c}\text { derivate } \\
\text { delle } \xi_{1}, \ldots, \xi_{n}\end{array}$ & funzioni $\alpha$ & $\begin{array}{r}\text { espressioni lineari omogenee } \\
\text { nelle derivate prime } \\
\text { delle } \omega_{1}, \ldots, \omega_{p}\end{array}$ \\
derivate & & funzioni $\alpha$ \\
del secondo ordine & & \\
\hline
\end{tabular}

Gli elementi che entrano a far parte della matrice sono quelli racchiusi entro il rettangolo marcato con linee più forti; esso è diviso in quattro regioni, per ciascuna delle quali è indicato l'elemento generico.

I determinanti che si possono ricavare da questa matrice sono formati o con sole funzioni $\alpha$, o con sole espressioni lineari omogenee nelle derivate delle $\omega_{s}$, o con entrambi questi elementi (nel qual caso o almeno una linea o almeno una colonna è co- 
stituita da elementi della stessa specie), o infine sono del tipo

\begin{tabular}{|c|c|}
\hline funzioni $\alpha$ & $\begin{array}{l}\text { espressioni } \beta \\
P\end{array}$ \\
\hline o & funzioni $\alpha$ \\
\hline
\end{tabular}

dove per brevitd si conviene di indicare con "espressione $\beta$ » ogni espressione lineare omogenea nelle derivate prime delle $\omega_{s}$.

E evidente che un tale determinante se il punto $P$ cade nella diagonale principale è eguale al prodotto di un determinante formato con sole funzioni $\alpha$ per un altro della stessa natura, se cade al disopra della diagonale è identicamente nullo, se cade sotto di essa è una espressione omogenea nelle derivate delle $\omega_{s}$ e quindi si annulla quando si supponga

(7)

$$
\omega_{\mathrm{I}}=\text { cost., } \ldots, \omega_{p}=\text { cost. }
$$

I determinanti formati con sole funzioni $\alpha$ debbono essere identicamente nulli perchè già si è osservato che non puó esistere tra le $\omega_{s}$ nessuna relazione di ordine zero; $\dot{\mathrm{s}}$ puó quindi concludere che se vi sono equazioni differenziali del primo ordine del tipo (4) a cui debbano soddisfare le funzioni $\omega_{s}$ una soluzione è sempre rappresentata dalle (7).

La estensione di questo risultato al caso in cui tra le equazioni differenziali che rimangono invarianti rispetto al gruppo (6) e che rivestono la forma (4) ve ne siano di ordine superiore al primo si puo ora ottenere facilmente, con un procedimento affatto analogo a quello testè seguito.

Se poniamo mente alla formola ricorrente dalla quale si deducono le variazioni delle derivate seconde, $\ldots, q^{\text {me }}$ delle $\omega_{s}$

$$
\Xi \frac{\partial}{\partial x_{h}}\left(\frac{\partial^{v_{1}+\cdots+\nu_{n}} \omega_{s}}{\partial x_{1}^{\nu_{1}} \ldots \partial x_{n}^{\nu_{n}}}\right)=\frac{\partial}{\partial x_{b}}\left(\Xi \frac{\partial^{v_{1}+\cdots+v_{n}} \omega_{s}}{\partial x_{1}^{\nu_{I}} \ldots \partial x_{n}^{\nu_{n}}}\right)-\sum_{i=1}^{n} \frac{\partial}{\partial x_{i}}\left(\frac{\partial^{\nu_{1}+\cdots+v_{n}} \omega_{s}}{\partial x_{1}^{\nu_{I}} \ldots \partial x_{n}^{\nu_{n}}}\right) \frac{\partial \xi_{i}}{\partial x_{b}}
$$

$\dot{e}$ facile anzitutto riconoscere che queste variazioni sono espresse in funzione lineare omogenea delle derivate prime, seconde, $\ldots$ delle $\xi_{1}, \ldots, \xi_{n}$; e precisamente la

$$
\Xi \frac{\partial^{v_{1}+\cdots+v_{n}} \omega_{s}}{\partial x_{1}^{\nu_{1}} \ldots \partial x_{n}^{v_{n}}} \quad\left(v_{1}+\cdots+v_{n}=q\right)
$$

è una espressione nella quale intervengono le derivate delle $\xi$ fino all'ordine $q+\mathrm{I}$, e nella quale le derivate di ordine $q+\mathrm{I}$ delle $\xi$ hanno per coefficienti le funzioni $\alpha_{i k s}$; quelle di ordine $q$ hanno per coefficienti delle funzioni lineari omogenee nelle derivate prime delle $\omega_{s}$; quelle di ordine $q-i$ hanno per coefficienti delle espressioni nelle quali 
intervengono le derivate prime, seconde, $\ldots(i+1)^{\text {me }}$ delle $\omega_{s}$. Tali espressioni si annullano ponendo $\omega_{1}=$ cost., $\ldots, \omega_{p}=$ cost. Conveniamo di chiamare "espressione $\beta$ " ogni espressione la quale si annulli facendo $\omega_{1}=$ cost., $\ldots, \omega_{p}=$ cost.. Allora la matrice $M$ dalla quale debbono ricavarsi tutti i sistemi differenziali invarianti di ordine $q$ puó essere schematicamente rappresentata come una matrice quadrata di ordine $q+\mathrm{I}$, i cui elementi $\varepsilon$ sono a loro volta matrici rettangolari. Le $\varepsilon$ della diagonale principale sono matrici i cui elementi sono funzioni $\alpha$; le $\varepsilon$ che si trovano al disotto della diagonale principale sono tutte costituite da elementi nulli, e le $\varepsilon$ che si trovano al disopra della diagonale principale sono costituite da espressioni $\beta$.

Quindi tutti i determinanti che si possono ricavare dalla matrice $M$ o sono identicamente nulli, o si annullano facendo le sostituzioni (7). Cosi il teorema che ogni tipo di Engel contiene un gruppo di PICARD risulta verificato per il caso di gruppi le cui equazioni di definizione sono del primo ordine.

3. Dopo aver cosi dimostrato l'esistenza, per ogni tipo di Engel, di un gruppo di PICARD, voglio ora indicare un procedimento molto semplice con il quale se ne possono formare le equazioni di definizione.

In una mia Memoria ${ }^{4}$ ) ho mostrato come si possa risalire dalla forma canonica di ENGEL per le equazioni delle trasformazioni infinitesime a quella per le trasformazioni finite. Se sono:

$$
\sum_{i \mid \alpha_{1}, \ldots, \alpha_{n}} \frac{\xi_{i j}^{\left(\alpha_{1} \ldots \alpha_{n}\right)}}{\alpha_{1} ! \ldots ! \alpha_{n} !} \lambda_{i \mid \alpha_{1}, \ldots, \alpha_{n \mid s}}\left[\omega_{1}\left(x_{1}, \ldots, x_{n}\right), \ldots, \omega_{p}\left(x_{1}, \ldots, x_{n}\right)\right]-\sum_{i=1}^{n} \xi_{i} \frac{\partial \omega_{s}}{\partial x_{i}}=0
$$

le equazioni delle trasformazioni infinitesime, bisogna trovare un sistema di funzioni $u_{1}, \ldots, u_{p}$ delle $y_{1}, \ldots, y_{n}, \ldots, y^{\left(\delta_{1}, \ldots, \delta_{n}\right)}, \ldots$ tale che sia:

$$
A_{i \mid \alpha_{1}, \ldots, \alpha_{n}}^{(m)}\left(u_{s}\right)=\lambda_{i\left|\alpha_{1}, \ldots, \alpha_{n \mid s}\right|}\left(u_{1}, \ldots, u_{p}\right)
$$

e tale inoltre che, ponendo $x_{1}, \ldots, x_{n}$ al posto di $y_{1}, \ldots, y_{n}$ rispettivamente, e per ciascuna delle $y_{i}^{\left(\delta_{1}, \ldots, \delta_{n}\right)}$ i valori che ne risultano, la $u_{s}$ si riduca ad $\omega_{s}\left(x_{1}, \ldots, x_{n}\right)$ per tutti i valori di $s$ da I a $p$.

Prescindiamo per il momento da questa seconda condizione; un sistema di funzioni delle $\ldots y_{i}^{\left(\delta_{1}, \ldots, \delta_{n}\right)} \ldots$ (le $y_{2}, \ldots, y_{n}$ non vi intervengono) tale che soddisfa alle (9) ci è dato dal sistema di invarianti indipendenti di un certo sottogruppo in $B_{m}$, intendendo indicare con $B_{m}$ il gruppo semplicemente transitivo che opera sulle derivate delle $y_{1}, \ldots, y_{n}$ rispetto alle $x_{1}, \ldots, x_{n}$ come la più generale trasformazione nelle $y_{1}, \ldots, y_{n}$ estesa fino all'ordine $m$.

Con $A_{m}$ si indica il reciproco di $B_{m}$, cioè il gruppo che opera sulle derivate delle $y_{1}, \ldots, y_{n}$ come la più generale trasformazione nelle $x_{1}, \ldots, x_{n}$ estesa fino all'ordine $m$.

Ogni gruppo isomorfo ad $A_{m}$, e tale è il gruppo che opera sulle $u_{1}, \ldots, u_{p}$ secondo le (9), si puó considerare infatti come l'indotto di $A_{m}$ sopra un sistema di

4) Sulla teoria dei gruppi infiniti continui [Annali di Matematica pura ed applicata, s. II, t. XXV (1897), pp. I79-217]. 
invarianti indipendenti di un sottogruppo di $B_{m}$. Se noi indichiamo questi invarianti con

(10)

le equazioni

(II)

$$
\begin{array}{cl}
\varphi_{s}\left(\ldots y_{i}^{\left(\delta_{1}, \ldots, \delta_{n}\right)} \ldots\right) & (s=\mathrm{I}, \ldots, p) \\
\psi_{s}=e_{s} & (s=\mathrm{I}, \ldots, p)
\end{array}
$$

ci definiscono un gruppo purchè si ponga per $e_{s}$ quel valore che assume $\varphi_{s}$ quando vi si faccia la sostituzione $y_{i}=x_{i},(i=\mathrm{I}, \ldots, n)$ cioè quando si pongano eguali a zero tutte le derivate che vi intervengono all'infuori delle $\frac{\partial y_{\mathrm{r}}}{\partial x_{\mathrm{I}}}, \ldots, \frac{\partial y_{n}}{\partial x_{n}}$ per le quali si sostituisce l'unità.

Le equazioni che definiscono le trasformazioni infinitesime di questo gruppo si ottengono ponendo $e_{s}$ al posto di $\omega_{s}$ nelle (8). I gruppi cosi definiti sono appunto i gruppi di PICARD dei quali nel paragrafo precedente si è constatata la esistenza quando $m=\mathrm{I}$. Il gruppo $B_{\mathrm{s}} \dot{\mathrm{e}}$ in tal caso uno dei due gruppi parametrici del gruppo lineare omogeneo in $n$ variabili, e gioverd per il nostro scopo ricordare, meno sommariamente di quanto sia stato fatto testè per il caso di $m$ qualunque, in qual modo vi si perviene.

Consideriamo una trasformazione infinitesima nelle $y_{x}, \ldots, y_{n}$

$$
Y f=n_{1}\left(y_{1}, \ldots, y_{n}\right) \frac{\partial f}{\partial y_{1}}+\cdots+n_{n}\left(y_{1}, \ldots, y_{n}\right) \frac{\partial f}{\partial y_{n}}
$$

ed estendiamola, coi noti procedimenti, per riguardo alle derivate prime delle $y_{1}, \ldots, y_{n}$ rispetto ad una nuova serie di variabili $x_{1}, \ldots, x_{n}$ non trasformate. Convertd ora introdurre le notazioni

$$
y_{i, k}=\frac{\partial y_{i}}{\partial x_{k}} ; \quad \eta_{i, k}=\frac{\partial \eta_{i}}{\partial y_{k}} \quad(i, k=\mathrm{I}, \ldots, n) .
$$

Si ha allora per la nostra $Y f$ estesa (che indicheremo con $Y^{(1)} f$ ) la espressione:

Le trasformazioni

$$
Y^{(1)} f=Y f+\sum_{i, k}^{n} n_{i, k}^{n} \sum_{i}^{1 \cdots n} y_{k, \mu} \frac{\partial f}{\partial y_{i, \mu}} .
$$

$$
B_{i, k}=\sum_{\mu}^{1 \ldots n} y_{k, \mu} \frac{\partial f}{\partial y_{i, \mu}}
$$

$(i, k=\mathbf{1}, \ldots, n)$

formano un gruppo $B_{1}$ parametrico di quello lineare omogeneo $L$ in $n$ variabili.

A due sottogruppi del gruppo $B_{1}$, equivalenti tra loro, corrisponde lo stesso gruppo di PICARD, e d'altra parte poichè il gruppo $B_{3}$ e quello lineare omogeneo sono isomorfi e quindi possiedono lo stesso gruppo aggiunto, a sottogruppi equivalenti entro $B_{\mathrm{I}}$ corrispondono sottogruppi equivalenti entro il gruppo lineare omogeneo $L$ e viceversa. Fra i gruppi di PICARD del primo ordine e i tipi di gruppi lineari omogenei si puo dunque stabilire una corrispondenza biunivoca; e per trovare tutti i gruppi di PICARD si adotterà il seguente procedimento :

Per ogni sottogruppo (tipico) in $L$ si consideri il corrispondente sottogruppo (tipico) $g$ in $B_{1}$; siano $\varphi_{1}, \ldots, \varphi_{p}$ i relativi invarianti; allora scrivendo le (II) si definisce un gruppo $G$ di PICARD del primo ordine.

La condizione necessaria e sufficiente affinchè la trasformazione infinitesima 
$Y f=\sum_{i=1}^{n} n_{i} \frac{\partial f}{\partial y_{i}}$ appartenga al gruppo $G$ definito dalle (I I) è che sia:
$Y\left(\varphi_{\mathrm{I}}\right)=0, \ldots, Y\left(\varphi_{p}\right)=0$

$$
Y\left(\varphi_{1}\right)=0, \ldots, Y\left(\varphi_{p}\right)=0
$$

e siccome le $\varphi$ non contengono le variabili $y_{\mathrm{r}}, \ldots, y_{n}$ dovrà essere:

$$
\sum_{i, k}^{1} \eta_{i, k}^{n} B_{i, k}\left(p_{s}\right)=0 \quad(s=1, \ldots, p) .
$$

Quindi la trasformazione infinitesima

$$
\sum_{i, k}^{1 \cdots n} n_{i, k} B_{i, k}
$$

per ogni sistema particolare di valori attribuito alle $y_{1}, \ldots, y_{n}$ dovrà appartenere al gruppo $g$, e perciò se noi indichiamo con

$$
\bar{B}_{1}, \ldots, \bar{B}_{n^{2}-p}
$$

le trasformazioni infinitesime indipendenti dal gruppo $g$ sarà identicamente:

$$
\sum_{i, k}^{\ldots n} n_{i, k} B_{i, k}=\sum_{\sigma=1}^{n^{2}=p} c_{\sigma}\left(y_{1}, \ldots, y_{n}\right) \bar{B}_{\sigma} .
$$

Di qui intanto si trae un altro procedimento per formare le equazioni di definizione del gruppo $G^{5}$ ). Siano

$$
\begin{aligned}
& l_{1}\left(\ldots a_{i, k} \ldots\right)=0 \\
& \ldots \ldots \\
& l_{p}\left(\ldots a_{i, k} \ldots\right)=0
\end{aligned}
$$

le relazioni lineari tra $\mathrm{i}$ parametri $a_{i, k}$ che definiscono il nostro sottogruppo $g$; sostituendo $n_{i, k}$ ad $a_{i, k}$ si ottengono le equazioni

$$
l_{s}\left(\ldots n_{i, k} \ldots\right)=0
$$$$
(s=\mathrm{I}, \ldots, p)
$$

che definiscono le trasformazioni infinitesime del gruppo $G$.

4. Mi propongo ora di applicare il metodo precedente allo studio dei gruppi infiniti. Poichè il gruppo lineare omogeneo si puó riferire isomorficamente a sè stesso, cosi anche l'isomorfismo tra questo gruppo e quello $B_{1}$ può presentarsi sotto due forme diverse. Nel seguito converrd far corrispondere alla trasformazione $x_{i} p_{k}$ del gruppo lineare omogeneo quella $B_{k, i}$ del gruppo $B_{\mathrm{s}}$. Inoltre per evitare ogni confusione scriveró sempre il gruppo $g$ lineare omogeneo nelle variabili $x_{\mathrm{r}}, \ldots, x_{n}$ ed il corrispondente gruppo $G$ nelle $y_{1}, \ldots, y_{n}$.

Cominciamo con lo studio di quei gruppi che corrispondono ai gruppi lineari omogenei semplicemente infiniti. Sia una trasformazione infinitesima

$$
X=\sum_{i, k}^{1 \cdot n} a_{i, k} x_{i} p_{k}
$$

5) Cfr. PiCard, Sur une gineralisation des équations de la théorie des fonctions d'une variable complexe [Comptes rendus hebdomadaires des séances de l'Académie des Sciences (Paris), t. CXII (I89I), pp. I399-1403]; Sur certains systèmes d'équations aux dérivées partielles généralisant les équations de la théorie des fonctions d'une variable complexe [Journal de Mathématiques pures et appliquées, IV ${ }^{\mathrm{e}}$ série, t. VIII (I892), pp. 2I 7-232]. 
alla quale dunque corrisponde, per le convenzioni fatte, la trasformazione $\sum_{i, k}^{r} a_{i, k} B_{k, i}$ di $B_{\mathrm{r}}$.

$\mathrm{La}$ (13) diventa:

$$
\sum_{i, k}^{\mathrm{r} \cdots n} n_{i, k} B_{i, k}=c\left(y_{1}, \ldots, y_{n}\right) \sum_{i, k}^{\mathrm{r} \cdots n} a_{i, k} B_{k, i}
$$

quindi si risolve nelle $n^{2}$ equazioni:

$$
n_{i, k}=c\left(y_{1}, \ldots, y_{n}\right) a_{k, i} .
$$

Le condizioni di integrabilità di questo sistema ci forniscono le equazioni:

$$
\frac{\partial c}{\partial y_{\mu}} a_{k, i}=\frac{\partial c}{\partial y_{k}} a_{\mu, i}
$$

Si possono distinguere due casi:

I) Tutte le derivate della $c$ sono nulle, cioè $c$ è una costante. Allora dalle (I4) si ricava, integrando:

$$
n_{i}=c \sum a_{k, i} y_{k}+c_{i}
$$

e quindi il gruppo $G$ è $\infty^{n+1}$ e la sua trasformazione infinitesima generica è composta oltre che con le traslazioni, con la sola $X$ (scritta nelle variabili $y$ ).

II) Se non tutte le derivate della $c$ sono nulle, allora deve essere

$$
\frac{a_{k, 1}}{a_{n, 1}}=\frac{a_{k, 2}}{a_{n, 2}}=\cdots=\frac{a_{k, n}}{a_{n, n}} \quad(k=1, \ldots, n),
$$

quindi la trasformazione $X$ è del tipo

$$
\left(\alpha_{1} x_{1}+\cdots+\alpha_{n} x_{n}\right)\left(\beta_{1} p_{1}+\cdots+\beta_{n} p_{n}\right) .
$$

Le (IS) diventano

quindi

$$
\frac{\partial c}{\partial y_{\mu}} \alpha_{k}=\frac{\partial c}{\partial y_{k}} \alpha_{\mu}
$$

$$
c=\Phi^{\prime}\left(\alpha_{1} y_{1}+\cdots+\alpha_{n} y_{n}\right),
$$

essendo $\Phi^{\prime}$ il simbolo di una funzione arbitraria, e dalle (I4) si trae:

$$
n_{i}=\Phi\left(\alpha_{1} y_{1}+\cdots+\alpha_{n} y_{n}\right) \beta_{i}+c_{i} \quad(i=1, \ldots, n) .
$$

Il gruppo $G$ è quindi infinito, e la sua trasformazione generica a prescindere dalle traslazioni è del tipo

$$
\Phi\left(x_{1} y_{1}+\cdots+\alpha_{n} y_{n}\right)\left(\beta_{1} p_{1}+\cdots+\beta_{n} p_{n}\right),
$$

essendo $\Phi$ il simbolo di una funzione arbitraria.

Teorema I. - Ad ogni trasformazione lineare omogenea $X$ si puo far corrispondere un gruppo $G$ contenente tutte le traslazioni.

Se la trasformazione $X$ è del tipo $l T$, essendo $l$ una funzione lineare omogenea $e T$ una traslazione, il gruppo $G$ che vi corrisponde è infinito, e le sue trasformazioni infinitesime, a parte un termine traslatorio, sono del tipo

$$
\varphi(l) . T \quad(\varphi \text { funzione arbitraria). }
$$


Se la trasformazione $X$ non è del tipo $l T$, il gruppo $G \dot{e} \infty^{n+1}$ e contiene, oltre le traslazioni, la $X$ stessa.

EsEMPI. - I) La trasformazione $X$ sia $x_{k} p_{i}$. Secondo le nostre convenzioni, per cercare il gruppo $G$ corrispondente si debbono calcolare anzitutto gli invarianti di

Si trovano cosi le funzioni:

$$
B_{i, k}=\sum_{\mu=1}^{n} y_{k, \mu} \frac{\partial f}{\partial y_{i, \mu}} \text {. }
$$

$$
y_{k, k} y_{i, \mu}-y_{k, \mu} y_{i, k} \quad\left(\begin{array}{l}
l=\mathrm{I}, \ldots, i-\mathrm{I}, i+\mathrm{I}, \ldots, n \\
\mu=\mathrm{I}, \ldots, n
\end{array}\right) .
$$

Il sistema delle equazioni di definizione si ottiene secondo le (I I) eguagliando ciascuna di queste funzioni al valore che essa assume quando si faccia $y_{1}=x_{1}, \ldots, y_{n}=x_{n}$. Si trova cosi :

$$
\begin{array}{llll}
\frac{\partial y_{l}}{\partial x_{\mu}}=\mathrm{o} & (l \neq \mu, i) ; & \frac{\partial y_{l}}{\partial x_{l}}=\mathrm{I} & (l \neq i) \\
\frac{\partial y_{i}}{\partial x_{\mu}}=\mathrm{o} & (i \neq \mu, \mu \neq k) ; & \frac{\partial y_{i}}{\partial x_{i}}=\mathrm{I} . &
\end{array}
$$

Integrando si ha:

$$
\begin{aligned}
& y_{l}=x_{l}+c_{l} \\
& y_{i}=x_{i}+c\left(x_{k}\right) ;
\end{aligned} \quad(l=\mathrm{r}, \ldots, i-\mathrm{r}, i+\mathrm{I}, \ldots, n),
$$

il gruppo $G$ è dunque bene quello

come indica il teorema.

$$
q_{\mathrm{r}}, \ldots, q_{n}, \xi\left(y_{k}\right) q_{i}
$$

2) Alla trasformazione $x_{k} p_{k}$ corrisponde il gruppo infinito

$$
q_{1}, \ldots, q_{n}, \xi\left(y_{k}\right) q_{k}
$$

3) Alla trasformazione $x_{1} p_{1}+\cdots+x_{n} p_{n}$ corrisponde il gruppo finito $\infty^{n+1}$

$$
q_{1}, \ldots, q_{n}, y_{1} q_{1}+\cdots+y_{n} q_{n}
$$

Tanto $i$ gruppi finiti quanto $i$ gruppi infiniti che si sono incontrati in questo paragrafo hanno in comune la proprietà di possedere nell'intorno della origine una ed una sola trasformazione indipendente del primo ordine. Proponiamoci ora questo problema: dato un gruppo del quale si sa che contiene nell'intorno di un punto generico (assunto ad origine delle coordinate) $n$ trasformazioni indipendenti di ordine zero, ed una sola trasformazione indipendente del primo ordine

$$
L=\sum_{\mu, i}^{1 \cdots n} a_{\mu, i} y_{\mu} q_{i}+\cdots,
$$

vediamo quante e quali trasformazioni di ordine superiore al primo esso puo contenere. 
Se una trasformazione del secondo ordine è rappresentata da

$$
\sum_{i=1}^{n} n_{i} q_{i}+\cdots
$$

dove $\mathrm{i}$ termini omessi sono di ordine superiore al secondo, sard certamente

$$
\sum_{i=1}^{n} n_{i, k} q_{i}+\cdots
$$

una trasformazione del primo ordine, o di ordine superiore, appartenente al gruppo, cioè sarà

$$
\sum_{i=1}^{n} n_{i, k} q_{i}=\rho_{k} \sum_{\mu, i}^{\mathrm{r} \ldots n} a_{\mu, i} y_{\mu} q_{i} .
$$

Queste si risolvono nelle $n^{2}$ equazioni

$$
n_{i, k}=\rho_{k} \sum_{\mu=1}^{n} a_{\mu, i} y_{\mu}
$$

che forniscono le condizioni di integrabilità:

$$
p_{k} a_{\mu, i}=p_{\mu} a_{k, i}
$$

$(i, k, \mu=\mathrm{r}, \ldots, n)$.

Confrontiamo queste con le (I5); anche qui possono presentarsi due casi: o tutte le $\rho$ sono nulle, e allora il gruppo non contiene nessuna trasformazione di ordine superiore al primo, o, se il gruppo ne contiene di tali, certamente la sua trasformazione del primo ordine è del tipo $l T+\cdots$, dove

$$
l=\sum_{\mu=1}^{n} p_{\mu} y_{\mu}, \quad T=\sum_{i=1}^{n} \beta_{i} q_{i} .
$$

Le (17) allora ci ddnno:

$$
\frac{\partial n_{i}}{\partial y_{k}}=\frac{\partial l}{\partial y_{k}} \cdot l \cdot \beta_{i}
$$

e se ne ricava, poichè le $n_{1}, \ldots, n_{n}$ sono funzioni omogenee delle $y_{1}, \ldots, y_{n}$,

$$
\sum_{i=1}^{n} n_{i} q_{i}=\frac{l^{2}}{2} \cdot T \text {. }
$$

Se fosse dimostrato che le trasformazioni di ordine $m$ sono del tipo

$$
l^{m} T+\cdots,
$$

se ne potrebbe subito dedurre che quelle di ordine $m+\mathrm{r}$ sono del tipo

$$
l^{m+1} T+\cdots \text {. }
$$

Basta infatti esprimere che la parentesi fra una trasformazione di ordine $m+\mathrm{I}$ ed una qualunque trasformazione di ordine zero è una trasformazione di ordine $\supseteq m$; se ne ricavano tali equazioni che dimostrano la proprietà cercata, la quale essendo vera per $m=2$, è vera dunque in generale. Per ogni ordine esiste pertanto al più una sola trasformazione indipendente, e, a meno che sia

$$
T(l)=o
$$


non possono esservi nel gruppo trasformazioni di ordine superiore al secondo, se esso è finito.

Nel caso particolare dei gruppi di Picard, quando cioè le trasformazioni di ordine zero sono traslazioni, possiamo giungere ad un risultato più preciso, dimostrando che ogni altra trasformazione è del tipo $\varphi(l) T$. Supponiamo infatti che considerando i successivi termini nello sviluppo delle trasformazioni del primo, secondo, ... ordine:

$$
\begin{array}{ll}
X_{1}=l T+u_{1,2}+u_{1,3}+\cdots+u_{1, m}+\cdots, \\
X_{2}= & l^{2} T+u_{2,3}+\cdots+u_{2, m}+\cdots, \\
X_{3}= & l^{3}+\cdots \\
X_{m}= & l^{m} T+\cdots,
\end{array}
$$

in cui le $u_{\mathrm{t}, i}, \ldots, u_{i-1, i}$ sono trasformazioni omogenee di grado $i$, si sia riusciti a dimostrare che ogni termine $u_{i, k}$ per $k \leq m$ (ed $i<k$ ) è del tipo $\alpha_{i, k} l^{k} T$, in cui $\alpha_{i, k}$ denota una costante.

$\mathrm{La}$ trasformazione

$$
\left(p_{k} X_{i}\right)-\frac{\partial l}{\partial y_{k}}\left[i X_{i-1}+\alpha_{i, i+1}(i+\mathrm{I}) X_{i}+\cdots+m \alpha_{i, m} X_{m-1}\right](k=1, \ldots, n ; i<m)
$$

appartiene al gruppo ed è dell'ordine $i$; il termine di grado $m$

$$
\frac{\partial u_{i, m+1}}{\partial y_{k}}-\frac{\partial l}{\partial y_{k}}\left(i \alpha_{i-1, m}+(i+\mathrm{I}) \alpha_{i, i+1} \alpha_{i, m}+\cdots+m \alpha_{i, m} \alpha_{m-1, m}\right) l^{m} T
$$

si deve dunque poter esprimere sotto la forma

$$
\left(A_{i} \alpha_{i, m}+\cdots+A_{m}\right) l^{m} T,
$$

in cui $A_{i}, \ldots, A_{m}$ sono costanti.

In conclusione dunque si ha

$$
\frac{\partial u_{i, m+1}}{\partial y_{k}}=B_{i, m+1 \mid k} l^{m} T
$$

in cui le $B_{i, m+1 k}$ sono costanti, ed integrando, tenendo conto delle condizioni di integrabilità:

Si ha dunque il

$$
u_{i, m+1}=\bar{B}_{i, m+1} l^{m+1} T \text {. }
$$

Teorema II. - Ogni gruppo che contenga il sottogruppo

$$
q_{1}, \ldots, q_{n}, \quad X=\sum a_{\mu, i} y_{\mu} q_{i}
$$

e nessuna trasformazione del primo ordine indipendente da $X$, contiene per ciascun $n u$ mero $m>$ I al piu una trasformazione di ordine $m$. Se vi sono trasformazioni di ordine $m>2$ il gruppo è infinito o abeliano, o possiede ambedue queste qualità. Quando il gruppo è infinito esso coincide con il gruppo $G$ del Teorema $I$.

Il gruppo $G$ pui pertanto definirsi come il pii ampio gruppo che contiene le (I8), e nessun'altra trasformazione del primo ordine indipendente da $X$.

5. Si conoscono tutti i tipi di gruppi semplicemente infiniti lineari omogenei in $n$ 
variabili; e noi potremmo quindi riferirci a tale determinazione per la ricerca di tutti $i$ gruppi $G$ che corrispondono ad una unica trasformazione infinitesima. Per quanto però riguarda $i$ gruppi infiniti questa ricerca si puó fare in modo molto più semplice, riferendosi al primo teorema or ora dimostrato.

Mediante una sostituzione lineare omogenea si può sempre ottenere che $l T$ acquisti la forma

$$
\left(a_{1} x_{1}+\cdots+a_{n} x_{n}\right) p_{1} \text {. }
$$

Se fosse $a_{\mathrm{r}}=0$, mediante una sostituzione del tipo

$$
\left\{\begin{array}{l}
x_{2}^{\prime}=b_{2} x_{2}+\cdots+b_{n} x_{n} \\
x_{i}^{\prime}=x_{i}
\end{array}\right.
$$

si puó ticondurre la (19) alla forma tipica $x_{2} p_{1}$.

Se fosse $a_{1} \neq 0$ dopo aver ridotta la (19) mediante una trasformazione della forma (20) a $\left(x_{2}+\alpha x_{1}\right) p_{1}$ si riduce ulteriormente questa al tipo $x_{1} p_{1}$ mediante la sostituzione:

$$
\begin{aligned}
& x_{2}^{\prime}=x_{2} \\
& x_{1}^{\prime}=x_{1}+\frac{1}{a} x_{2} .
\end{aligned}
$$

Osserviamo d'altra parte che i gruppi $G$ i quali corrispondono ad una unica trasformazione infinitesima sono caratterizzati dall'avere un sistema di $n^{2}-\mathrm{r}$ equazioni di definizione. Quindi :

TeOrema III. - I gruppi transitivi di PICARD, definiti da un sistema di $n^{2}-\mathrm{I}$ equazioni differenziali del primo ordine, possono essere finiti o infniti. I gruppi finiti sono ad $n+\mathrm{I}$ parametri e possono ricondursi alla forma tipica:

$$
\overline{q_{\mathrm{s}}, \ldots, q_{n}, L}
$$

in cui $L$ e una trasformazione infinitesima (tipica) lineare omogenea. I gruppi infiniti sono o del tipo:

o del tipo:

$$
q_{1}, \ldots, q_{n}, \quad \xi\left(y_{1}\right) q_{1}
$$

$$
q_{\mathrm{t}}, \ldots, q_{n}, \quad \xi\left(y_{2}\right) q_{\mathrm{r}}
$$

in cui la $\xi$ è ogni volta una funzione arbitraria dell'argomento.

6. Dobbiamo ora occuparci dei gruppi $G$ che corrispondono ai gruppi lineari $\infty^{r}$ per $\underset{\text { Siano }}{r>}$

$$
L_{s}=\sum_{i, k}^{r \cdots n} a_{s, i, k} x_{i} p_{k} \quad(s=1, \ldots, r)
$$

le trasformazioni infinitesime indipendenti di un gruppo $r^{\text {plo }}$ lineare omogeneo. Il gruppo $G$ corrispondente contiene intanto, oltre le traslazioni, certamente anche le $r$ trasformazioni (24) (scritte nelle variabili $y_{1}, \ldots, y_{n}$ ). Infatti per le (13) dovrà essere

$$
\sum_{k, i}^{\mathrm{r} \ldots n} n_{k, i} B_{k, i}=\sum_{s=1}^{r} c_{s}\left(y_{1}, \ldots, y_{n}\right) \sum_{i, k}^{1 \cdots n} a_{s, i, k} B_{k, i}
$$


quindi

$$
\eta_{k, i}=\sum_{s=1}^{r} c_{s}\left(y_{1}, \ldots, y_{n}\right) a_{s, i, k}
$$$$
(k, i=\mathrm{I}, \ldots, n) \text {. }
$$

Una soluzione dalle (25) si ottiene in ogni caso ponendo:

i valori corrispondenti delle $c$ sono :

$$
n_{k}=\sum_{i=1}^{n} a_{s, i, k} y_{i}
$$

$$
(k=1, \ldots, n),
$$

$$
c_{s}=\mathrm{I} ; \quad c_{\sigma}=0 \quad(\sigma=\mathrm{r}, \ldots, s-\mathrm{I}, s+\mathrm{I}, \ldots, n) .
$$

$\mathrm{Si}$ vede dunque che il gruppo $G$ contiene le $r$ trasformazioni infinitesime

$$
L_{s}=\sum_{i, k}^{1 \ldots n} a_{s, i, k} y_{i} q_{k} \quad(s=\mathrm{I}, \ldots, r) .
$$

Ma si puó anche dimostrare che non vi sono nel gruppo $G$ altre trasformazioni del primo ordine indipendenti $\mathrm{da}$ queste.

Infatti sia $Y$ una qualunque trasformazione del primo ordine del gruppo $G$.

$$
Y=\sum_{i, k}^{1 \ldots n} b_{i, k} y_{i} q_{k}+\sum_{l, i, k}^{\mathrm{r} \dddot{n}} b_{l, i, k} y_{l} y_{i} q_{k}+\cdots
$$

Portando i valori delle $r_{k, i}$ corrispondenti nelle (25) si avrebbe

$$
b_{i, k}+\sum_{l=1}^{n} b_{l, i, k} y_{l}+\cdots=\sum_{s=1}^{r} c_{s} a_{s, i, k}
$$

e dovendo queste essere soddisfatte identicamente, e quindi anche per

resta :

$$
y_{\mathrm{x}}=y_{2}=\cdots=y_{n}=0
$$

$$
b_{i, k}=\sum_{s=1}^{r} c_{s}(\mathrm{o}, \ldots, 0) a_{s, i, k}
$$

e perciò la trasformazione

$$
Y-\sum_{s=1}^{r} c_{s}(0, \ldots, 0) L_{s}
$$

è di ordine superiore al primo, ciò che appunto si trattava di dimostrare.

$\mathrm{Si}$ ha dunque :

Teorema IV. - Ad ogni gruppo lineare omogeneo $\left(L_{\mathrm{s}}, \ldots, L_{r}\right)$ si puo far corrispondere un gruppo $G$ il quale contiene:

a) tutte le traslazioni

b) le trasformazioni $L_{1}, \ldots, L_{r}$,

c) eventualmente altre trasformazioni di ordine superiore al primo, nell'intorno dellorigine.

Non si puó concludere, diversamente da quanto si osserva per il caso $r=\mathrm{I}$, che quando intervengono trasformazioni di ordine superiore al primo il gruppo $G$ è infinito. Un esempio sard sufficiente a renderne ragione.

Nel gruppo lineare omogeneo generale in tre variabili, la cui trasformazione infini- 
tesima generica è

$$
\left(a_{1,1} x+a_{1,2} y+a_{1,3} z\right) p+\left(a_{2, \mathrm{~L}} x+a_{2,2} y+a_{2,3} z\right) q+\left(a_{3,1} x+a_{3,2} y+a_{3,3} z\right) r
$$

si ottiene un sottogruppo $\infty^{4}$, assoggettando i parametri $a_{i, k}$ alle seguenti condizioni :

$$
\left\{\begin{array}{c}
a_{\mathrm{1}, 3}=a_{2,1}, \quad a_{3,2}=0, \quad a_{3, \mathrm{1}}=a_{\mathrm{t}, 2}, \quad a_{2,3}=0 \\
a_{2,2}+a_{3,3}-2 a_{\mathrm{t}, \mathrm{r}}=0 ;
\end{array}\right.
$$

per trasformazioni infinitesime indipendenti del gruppo si possono prendere le

$$
[x p+y q+z r, \quad z p+x q, \quad y q-z r, \quad x r+y p] ;
$$

il gruppo $G$ corrispondente è definito dal sistema di equazioni

$$
\left\{\begin{array}{cc}
\frac{\partial \xi}{\partial z}=\frac{\partial n}{\partial x}, & \frac{\partial \xi}{\partial y}=\frac{\partial \zeta}{\partial x}, \quad \frac{\partial n}{\partial z}=0, \\
\frac{\partial n}{\partial y}+\frac{\partial \zeta}{\partial z}-2 \frac{\partial \xi}{\partial x}=0, & \frac{\partial \zeta}{\partial y}=0,
\end{array}\right.
$$

che si deducono dalle (26) sostituendo ad $a_{1,1}, a_{1,2}, a_{1,3}$ rispettivamente $\frac{\partial \xi}{\partial x}, \frac{\partial \xi}{\partial y}, \frac{\partial \xi}{\partial z}$; ad $a_{2,1}, a_{2,2}, a_{2,3}$ rispettivamente $\frac{\partial \eta}{\partial x}, \frac{\partial \eta}{\partial y}, \frac{\partial \eta}{\partial z}$ e infine ad $a_{3,1}, a_{3,2}, a_{3,3}$ le $\frac{\partial \zeta}{\partial x}, \frac{\partial \zeta}{\partial y}, \frac{\partial \zeta}{\partial z}$.

Il sistema (27) ci dimostra che il gruppo $G$ contiene oltre le traslazioni ed oltre le trasformazioni $\left(26^{*}\right)$, soltanto le tre trasformazioni del secondo ordine seguenti:

$$
\left\{\begin{array}{c}
2 x z p+x^{2} q+2 z^{2} r \\
2 x y p+x^{2} r+2 y^{2} q \\
\left(2 z y+x^{2}\right) p+2 x y q+2 x z r .
\end{array}\right.
$$

7. Per vedere ora l'intimo nesso che intercede tra i gruppi $g$ e $G$, conviene intro. durre una nuova nozione. Sia $X$ una trasformazione infinitesima di ordine $m$ nell'intorno della origine; formando la parentesi tra la $X$ ed una qualunque traslazione ne risulta una trasformazione di ordine $\supseteq m-$ I. Iterando questo procedimento $\mu$. volte si perviene, purchè $\mu$ sia convenientemente grande $(\supseteq m-1)$ ad una trasformazione del primo ordine, e combinando a sua volta questa una, due, ... volte con le traslazioni si ottengono sempre trasformazioni di ordine zero (o superiore).

Combinando dunque in tutti i modi possibili ed iteratamente la $X$ con le traslazioni si perviene in ultima analisi ad un certo numero di trasformazioni di ordine inferiore al secondo.

Se tutte le trasformazioni cosi dedotte dalla $X$ si presentano sotto la forma (29) $a_{i} p_{1}+\cdots+a_{n} p_{n}+b_{1} L_{1}+\cdots+b_{p} L_{p}+$ una trasformazione di ordine superiore al primo, in cui le $a_{1}, \ldots, a_{n}, b_{1}, \ldots, b_{p}$ sono costanti, $L_{1}, \ldots, L_{p}$ sono trasformazioni infinitesime indipendenti di un gruppo lineare omogeneo $g$, io diro che la trasformazione $X$ è congrua al gruppo $g$.

Si noti che: genee

$I^{0}$ Se la trasformazione $X$ si presenta come la somma di più trasformazioni omo-

$$
X=U_{m}+U_{m+1}+\cdots,
$$


in cui i coefficienti di $U_{m}$ sono funzioni omogenee di grado $m$, quelli di $U_{m+1}$ sono di grado $m+\mathrm{I}, \ldots$, anche le $U_{m}, U_{m+1}, \ldots$ sono separatamente congrue al gruppo $g$.

$2^{\mathrm{o}}$ Se $X$ ed $Y$ sono congrue al gruppo g, lo è anche ogni combinazione lineare $a X+b Y$, a coefficienti costanti $a, b$ comunque scelti.

$3^{\circ}$ Se $X$ ed $Y$ sono congrue al gruppo $g$, anche la trasformazione $(X Y)$ lo è.

Per la osservazione $\left.I^{2}\right)$ potremo limitarci a dimostrare questa proprietà sopra le trasformazioni omogenee.

Se $Y$ è del primo ordine e $\pi$ è una qualunque traslazione, dalla identità di JACOBI, formata con le tre trasformazioni $X, Y, \pi$, risulta anzitutto dimostrato il teorema per il caso in cui $X$ sia di ordine 2, e risulta poi per induzione dimostrato in ogni altro caso. E ancora dalla identità di JACOBI, in cui intervenga una traslazione, risulta dimostrato il teorema nel caso più generale in cui $X$ ed $Y$ siano trasformazioni di ordine qualunque.

Le trasformazioni congrue ad uno stesso gruppo lineare omogeneo $g$ formano dunque un gruppo, ed io voglio ora mostrare che questo gruppo è appunto quello che noi abbiam convenuto di indicare con $G$.

Il gruppo delle trasformazioni congrue è intanto, per il modo stesso della sua definizione, il più ampio fra tutti i gruppi la cui più generale trasformazione è nei termini di ordine zero ed uno del tipo (29), e siccome il gruppo $G$ è appunto uno di tali gruppi, esso è intanto certamente contenuto nel gruppo di tutte le trasformazioni congrue. Se ne fosse un effettivo sottogruppo i due sistemi di equazioni di definizione che corrispondono l'uno al gruppo $G$, l'altro al gruppo delle trasformazioni congrue, dovrebbero differire per il numero delle equazioni, e precisamente $G$ dovrebbe contenere un numero maggiore di equazioni di definizione. Ma ciò è impossibile rispetto a quelle del primo ordine, perchè $\mathrm{i}$ due gruppi contengono lo stesso numero di trasformazioni infinitesime indipendenti del primo ordine, nè puó essere nemmeno per quelle di ordine superiore, perchè queste rispetto a $G$ sono tutte ottenute per derivazione delle equazioni del primo ordine. Quindi i due gruppi coincidono. Si ha cosi il

Teorema V. - Dato un gruppo g lineare omogeneo

$$
\left[L_{1}, \ldots, L_{p}\right]
$$

si chiami congrua a g ogni trasformazione di ordine superiore al primo tale che non sia possibile per via di derivazioni (e conseguentemente di parentesi con $L_{\mathrm{x}}, \ldots, L_{\mathrm{p}}$ ) dedurne trasformazioni del primo ordine indipendenti dalle $L_{1}, \ldots, L_{p}$ stesse.

Le trasformazioni congrue a $g$ costituiscono, insieme con g stesso e con le traslazioni, un gruppo eventualmente infinito, il quale coincide con il gruppo $G$ del teorema $I V$.

Roma, maggio 1907.

P. MEDOLAGHI. 